\title{
Recent results from ATLAS on B Physics and Quarkonia
}

\author{
RWL Jones ${ }^{1}$ for the ATLAS Collaboration \\ ${ }^{1}$ Department of Physics, Lancaster University, Lancaster LA1 4YB, UK
}

\begin{abstract}
Recent results from the extensive programme of heavy flavour and onia studies in ATLAS are presented. These benefit from the very high integrated luminosity in the first running period at the LHC at 7 and $8 \mathrm{TeV}$, and some are now extended to include information from the latest $13 \mathrm{TeV}$ running.
\end{abstract}

\section{Introduction}

Heavy flavour physics in ATLAS [1] has both intrinsic interest as laboratory for the standard model and as a potential window into new physics. ATLAS has the advantage of high integrated luminosities and good muon triggers, and is particularly well suited for multi-muon final states. It also has excellent charged particle tracking and lifetime resolution, which has been further improved in 2015 by the addition of the insertable B-layer (IBL) [2], reducing from $100 \mathrm{fs}$ to $50 \mathrm{fs}$. The momentum resolution is less than $10 \%$ up to $1 \mathrm{TeV}$. The typical heavy flavour primary trigger require either two muons of at least $4 \mathrm{GeV}$ momentum transverse to the beam $\left(p_{T}\right)$ or else $p_{T}$ thresholds of 4 and $6 \mathrm{GeV}$ when rates must be reduced. Prescales are applied when necessary.

\section{Onium Production}

The foundation of many of the heavy flavour studies in ATLAS is onium reconstruction, and particularly the $\mathrm{J} / \psi$. ATLAS has extended its studies of $\mathrm{J} / \psi$ and $\psi(2 S)$ production from its studies at 7 and $8 \mathrm{TeV}$ centre of mass energies to include the new $13 \mathrm{TeV}$ data [3].

\section{$2.1 \mathrm{~J} / \psi$ and $\psi(2 S)$ production}

Both prompt and non-prompt production is studied. The two onia have individual strengths; the $\psi(2 S)$ has no significant feed-down from higher states, and allows the $J^{P C}=1^{--}$states to be studied. The $\mathrm{J} / \psi$, which is more plentiful, has contributions from $1^{--}$and $J^{++}$in comparable amounts.

The studies use $4 \mathrm{GeV}$ thresholds for both muons, and corrections are made for trigger and reconstruction efficiencies. The acceptance depends on the spin alignment; current measurements support the central assumption of no alignment. The prompt and non-prompt components are distinguished by constructing the pseudo-proper decay time using the transverse displacement of the the decay vertex from the interaction point and the $p_{T}$ of the onium. The prompt production is compared with nonrelativistic QCD predictions (NRQCD, see for example [4] and [5]), and good agreement is found 
across the wide range of $p_{T}$, with no dependence on rapidity, $y$. The non-prompt production is assumed to be dominated by onia from B-decays, and are compared to fixed order, next to leading logarithm (FONLL) predictions. Here, there is evidence that the $\psi(2 S)$ predictions tend to overestimate the differential cross sections. Thus far, double differential cross-sections are available from the $13 \mathrm{TeV}$ data [6]. The results now span centre of mass energies from 2.76 to $13 \mathrm{TeV}$ within one experiment and with self-consistent analyses. Strikingly, there is no significant change in the the fraction of non-prompt production with either rapidity or centre of mass energy, as illustrated in figure 1 and 2.

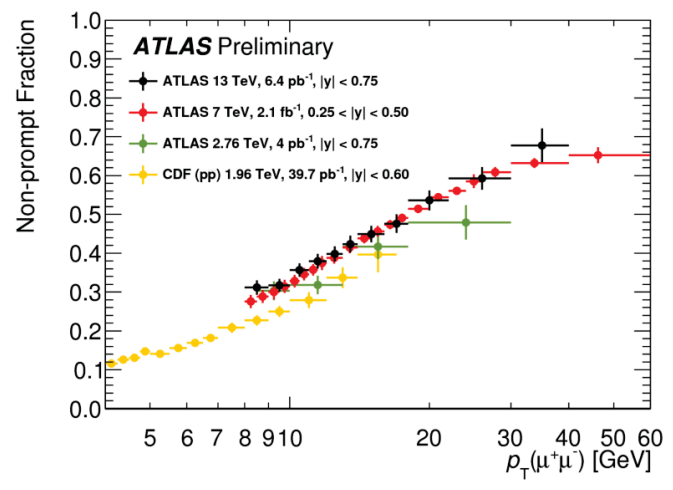

Figure 1. The fraction of prompt $\mathrm{J} / \psi$ production as a function of $p_{T}$ for different centre of mass energies observed in ATLAS $p p$ collisions and in CDF $p \bar{p}$ collisions [6].

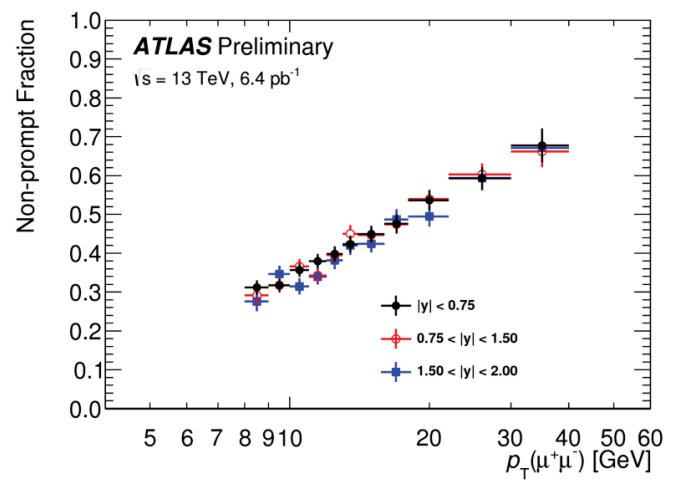

Figure 2. The fraction of prompt $\mathrm{J} / \psi$ production as a function of $p_{T}$ for different rapidity $y$ intervals as observed in the $13 \mathrm{TeV}$ data [6].

\subsection{Excited $B_{c}$ production}

With the first observation of the $\chi_{b}(3 P)$ in 2012 [7], ATLAS showed its potential for new particle discoveries. The ATLAS search has continued with the observation of a state consistent with an 
excited $B_{c}$ state [8]. This was observed both in the $7 \mathrm{TeV}$ and $8 \mathrm{TeV}$ data. The $B_{c}^{ \pm}$was reconstructed from its decay to $\mathrm{J} / \psi(\mu \mu) \pi^{ \pm}$. Opposite charged particles (where the particles are inconsistent with being muons and consistent with originating from the same interaction as the $B_{c}^{ \pm}$are added in pairs to the $B_{c}^{ \pm}$, and the mass difference spectrum $Q=m\left(B_{c}^{ \pm} \pi^{+} \pi^{-}\right)-m\left(B_{c}^{ \pm}-2 \cdot m\left(\pi^{ \pm}\right)\right.$is constructed. A new structure is observed in both samples, consistent with predictions for a $B_{c}^{ \pm}(2 S)$ meson. The total significance for the new particle is $5.2 \sigma$ when look-elsewhere effects are taken into account.

\subsection{Search for hidden beauty in $\Upsilon(1 S) \pi \pi$}

Searches have also been made for hidden beauty states in the $\Upsilon(1 S) \pi \pi$ decay mode (where the $\Upsilon(2 S$ ) and $\Upsilon(3 S)$ regions are excluded) [9]. This has resulted in the most sensitive $X_{b}$ production search in the mass range $m(\Upsilon(1 S) \pi \pi)>10.1 \mathrm{GeV}$. This state would be an analogue to the $X(3872)$ narrow resonance observed in the charmonium sector, which is still an unresolved mystery. This has revealed no evidence for the $\Upsilon\left(1^{3} D_{J}\right), \Upsilon(10860)$ and $\Upsilon(11020)$ states, or in general for a beauty analogue to the $X(3872)$.

\subsection{Charmonia in association with Vector Bosons}

The high available statistics combined with the tools for studying charmonia allow for the study of associated production between intermediate vector bosons and charmonia. These allow for test of QCD predictions at the perturbative/non-perturbative boundary. Anomalous rates provide evidence for processes beyond the standard model from charged Higgs decays, light scalars etc. For the prompt associated production, the colour octet and colour singlet contributions are uncertain and can be probed. Similarly, the contributions from single parton scattering and double parton scattering (where the two objects come from separate processes) are not well understood, and can be disentangled. The latter can be an important uncertainty for other studies, such as the measurement of the Higgs couplings.

ATLAS has measured the associated production of a $W^{ \pm}$and a prompt $\mathrm{J} / \psi$ at $7 \mathrm{TeV}$ [10]. It is found that the single parton scattering dominates at low $p_{T}$ of the $\mathrm{J} / \psi$, although considerable double parton scattering is evident. The NLO colour singlet predictions are well below the observed rates, while the colour octet has a considerably smaller colour octet contribution from non-relativistic QCD (NRQCD). The combined theory predictions fall short of the observations.

ATLAS has also measured the associated production of a $Z$ and a prompt $\mathrm{J} / \psi$ at $8 \mathrm{TeV}$ [11]. Both prompt and non-prompt $\mathrm{J} / \psi$ have been studied, with unambiguous observations in both modes, and both the azimuthal angle between the $Z$ and the $\mathrm{J} / \psi, \Delta \phi$, and the differential cross-section as a function of the $p_{T}$ of the $\mathrm{J} / \psi$ have been studied. The smallest bin of $\delta \phi$ for the prompt case allows the maximum allowed rate for double parton scattering to be set. Double parton scattering makes up $29 \pm 9 \%$ of the prompt production but only $8 \pm 2 \%$ of the non-prompt. The $p_{T}$ spectra again exceed the combined colour octet and colour singlet predictions. The associated prompt production represents $(63 \pm 13 \pm 5 \pm 10) \times 10^{-7}$ of the inclusive $Z$ production, and the non-prompt represents $(102 \pm 15 \pm 5 \pm 3) \times 10^{-7}$ of the inclusive $Z$ production both in the range $8.5<p_{T}(J / \psi)<100 \mathrm{GeV}$ and $|y(J / \psi)|<2.1$.

\section{B physics measurements}

ATLAS has a large and established B-physics programme, with many recent additions making use of the full run 1 statistics. The recent results involve investigations of new decay channels, the decay properties and the investigation of potential $\mathrm{CP}$ violation and new physics using specific channels. 


\subsection{Decays of the $B^{ \pm}$}

ATLAS has produced recent results on the decay of the $B_{c}^{+}$(and its charge conjugate, implied), in the decay mode $B_{c}^{+} \rightarrow J / \psi D_{s}^{+}$and $B_{c}^{+} \rightarrow J / \psi D_{s}^{*+}$ [12]. The $D_{s}$ is reconstructed in its decay to $\phi(K K) \pi^{+}$. The equivalent $D_{s}^{*+}$ is not explicitly reconstructed using the soft $\pi^{0}$ or photon from the decay, but rather using a cascade vertex fit, using pointing, mass and vertex constraints. A two-dimensional fit is then performed to the fitted mass and helicity angle to measure the branching ratios in each channel and the relative transverse polarisation

$$
\Gamma_{ \pm \pm}\left(B_{c}^{+} \rightarrow J / \psi D_{s}^{*+}\right) / \Gamma\left(B_{c}^{+} \rightarrow J / \psi D_{s}^{*+}\right)=0.38 \pm 0.23 \text { (stat.) } \pm 0.07 \text { (syst.) }
$$

this is in good agreement with LHCb and previous measurements. The relative branching ratio for $B_{c}^{+} \rightarrow J / \psi D_{s}^{+}$and $B_{c}^{+} \rightarrow J / \psi D_{s}^{*+}$ relative to $B_{c}^{+} \rightarrow J / \psi \pi^{+}$are higher than in pre-LHC measurements, but for the $B_{c}^{+} \rightarrow J / \psi D_{s}^{+}$the LHCb measurement agrees with ATLAS; no LHCb result exists as yet for the other case.

\subsection{Observation of $\Lambda_{B} \psi(2 S) \Lambda$}

ATLAS has made the first observation of the decay mode $\Lambda_{B} \psi(2 S) \Lambda$ using its $8 \mathrm{TeV}$ data [13]. This was measured in the kinematic range $p_{T}\left(\Lambda_{b}^{0}\right)>10 \mathrm{GeV}$ and $\mid \eta\left(\left(\Lambda_{b}^{0}\right) \mid<2.1\right.$. The following relative branching fraction has been measured:

$$
\left.\frac{\Gamma\left(\Lambda_{B}^{0} \rightarrow \psi(2 S) \Lambda\right)}{\Gamma\left(\Lambda_{B}^{0} \rightarrow J / \psi \Lambda\right)}=0.501 \pm 0.033 \text { (stat.) } \pm 0.016 \text { (syst. }\right) \pm 0.011(\mathcal{B})
$$

here, $\mathcal{B}$ is the uncertainty from the branching fraction for each of the onium states to muons.

\subsection{New measurements using the decay $B_{s} \rightarrow J / \psi \phi$}

ATLAS has presented a new determination of the CP-violating phase, $\phi_{s}$, and the $B_{s}$ meson width difference using the decays $B_{s} \rightarrow J / \psi \phi$ [14]. This analysis uses both the $7 \mathrm{TeV}$ and $8 \mathrm{TeV}$ datasets, which have then been combined. The $\mathrm{CP}$ violating phase is potentially sensitive to new physics contributions, while the width difference (measuring the lifetime difference between the CP even and odd eigenstates) is less sensitive to new physics but constrains the phenomenological models. As the decay mode chosen has a vector-vector final state, a time dependent flavour tagged analysis allows the two eigenstates to be resolved, characterised by three decay angles in the transversity basis. The analysis has the advantage of high statistics and relatively easy triggering, and is free of lifetime cuts. In this case, the term charge tagging refers to a determination if the initial quark leading to $B_{s}$ decay was a quark or an antiquark. Opposite side tagging is used, which means the initial quark is inferred from the decay of the other associated B-hadron created in the event. The data is calibrated from the data using the self-tagging decay $B^{ \pm} \rightarrow J / \psi K^{ \pm}$.

The final analysis uses an unbinned maximum likelihood fit to nine physics parameters, and the three of most physics interest are shown in table 1 . Both $\phi_{s}$ and $\Delta \Gamma_{s}$ are consistent with other measurements and with the standard model prediction, as illustrated for the combined result in figure 3 . With the greatly improved lifetime resolution in the new run, these results can be greatly improved, limited only by the available trigger bandwidth for the foreseeable future. 
Table 1. The three most relevant physics parameters of the nine fitted in the decay using the unbinned maxiumum likelihood fit to the 7 and $8 \mathrm{TeV}$ data [14]; the results are from the paper as submitted for publication and so a minor modification from those presented at the conference.

\begin{tabular}{lllll}
\hline Parameter & Value & Stat. & Syst. & units \\
\hline$\phi_{s}$ & -0.098 & 0.084 & 0.040 & $\mathrm{rad}$ \\
$\Delta \Gamma_{s}$ & 0.083 & 0.011 & 0.007 & $\mathrm{ps}^{-1}$ \\
$\Gamma_{s}$ & 0.677 & 0.003 & 0.003 & $\mathrm{ps}^{-1}$ \\
\hline
\end{tabular}

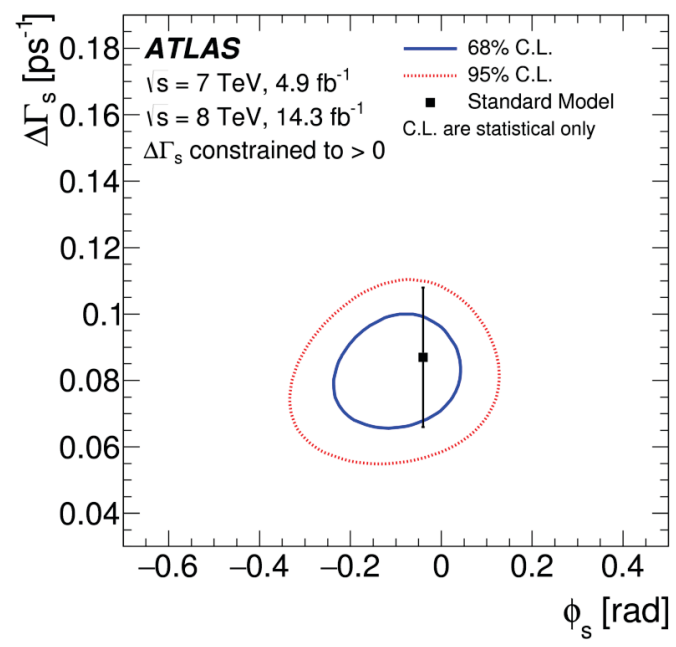

Figure 3. The combined 7 and $8 \mathrm{TeV}$ results for $\phi_{s}$ and $\Delta \Gamma$ along with the Standard Model expectation [14].

\section{References}

[1] ATLAS Collaboration, JINST 3, S08003 (2008)

[2] ATLAS Collaboration, CERN-LHCC-2012-009 (2012) https://cds.cern.ch/record/1451888

[3] ATLAS Collaboration, CERN-PH-EP-2015-292 (2015) arXiv:1512.03657 [hep-ex]

[4] G.T. Bodwin, E. Braaten and G. P. Lepage, Phys. Rev. D51, 1125 (1995)

[5] M. Butenschoen and B. A. Kniehl, Nucl. Phys. Proc. Suppl. 222-224, 151 (2012)

[6] ATLAS Collaboration, ATLAS-CONF-2015-030 (2015) https://cds.cern.ch/record/2037967

[7] ATLAS Collaboration, Phys. Rev. Lett. 108, 152001 (2012)

[8] ATLAS Collaboration, Phys. Rev. Lett. 113, 212004 (2014)

[9] ATLAS Collaboration, Phys. Lett. B740, 199 (2015)

[10] ATLAS Collaboration, JHEP 04, 172 (2014)

[11] ATLAS Collaboration, Eur. Phys. J. C75, 229 (2015)

[12] ATLAS Collaboration, Eur. Phys. J. C 761 (2016)

[13] ATLAS Collaboration, Phys. Lett. B 751, 63 (2015)

[14] The ATLAS Collaboration, CERN-PH-EP-2015-166 (2015) arXiv:1601.03297 [hep-ex] 